\title{
Guidelines for regional advisers on consultant posts in forensic psychiatry
}

\author{
Forensic Psychiatry Section
}

\section{Introduction}

Forensic psychiatry is a relatively new specialty of psychiatry which has developed very rapidly since 1975 with the development of the regional secure unit programme. Forensic psychiatrists are responsible for the assessment and management of some of the most seriously disturbed, disruptive and dangerous people presenting to psychiatry. Most psychiatrists in other psychiatric specialties evaluate and treat offender patients, but the core of the average forensic psychiatrist's work is with people who have seriously offended in the context of their mental disorder. The forensic psychiatrist must be equally skilled in providing treatment within secure conditions and in open facilities or in the community. In practice, there are at least two levels of security in which forensic psychiatrists may work within the health service: in maximum security (Special) hospitals for England and Wales (Ashworth, Broadmoor and Rampton Hospitals) and, in Scotland, Carstairs, and in the settings of medium security offered now by most regional health authorities in purpose-built secure units. In addition, forensic psychiatrists may treat some inpatients in open psychiatric units, and will see out-patients in hospitals, health centres, probation offices and other settings in the community. They may also work within the Prison Medical Service, usually on a sessional basis while holding a substantive health service post.

The specifications for individual consultant posts in forensic psychiatry should be tailored to suit local needs.

\section{Sources of further advice}

Further advice on these guidelines may be obtained from regional representatives of the Forensic Section of the College and regional advisers are expected to consult these colleagues with regard to job descriptions. A list of regional representatives may be obtained from the College. Additionally, regional advisers may wish to consult with local specialty tutors in forensic psychiatry.

\section{Features of posts}

\section{(a) Clinical facilities}

There should be a clear statement on the in-patient facilities attached to the post.

In the setting of regional forensic psychiatric services based upon regional (medium) secure units, there is a necessity to employ at least two consultant forensic psychiatrists per in-patient unit. This necessity arises from a number of factors, including the diverse demands of forensic psychiatry, the stresses involved in working overwhelmingly in the field of antisocial activity and the intensive therapeutic activity necessary. The length and intensity of commitment required for substantial numbers of demanding, difficult and dangerous patients require a wide network of trusted expert colleagues to provide opportunities for continuing advice and support. Thus a consultant forensic psychiatrist should not be expected to work in isolation.

The College recommended in 1975, as a minimum provision, that two consultants should be responsible for 25 medium secure in-patient beds, which with government norms of 20 beds per million of population equates to one consultant per 625,000 of the population. Subsequent experience of the running of regional forensic psychiatric services based upon regional secure units, whose bed numbers are calculated as above, confirms that this figure is the minimum consultant resource necessary. Furthermore, it makes no allowance for the special needs of some areas - e.g. higher referral rates in some inner city areas, and the perceived problems of smaller units or areas of widespread geographical distribution, where a single consultant service can be very vulnerable. Local factors such as these should be taken into consideration in evaluating an individual post.

In the setting of a special hospital, the standard consultant:patient ratio is currently agreed at $\mathbf{5 0}$ in-patients per consultant forensic psychiatrist. Additionally, it is recommended that special hospital consultants should each have two sessions working outside the maximum secure perimeter, in the setting of a regional secure unit, or other relevant activity, for example in an academic unit, with the aim of 
further reducing professional isolation and providing continuity of care for former special hospital patients. In these circumstances, the special hospital consultant caseload should ideally be reduced from 50 to 40 in-patients per consultant.

There should be a statement of the out-patient facilities provided, either at the secure unit or in a psychiatric hospital or district general hospital setting.

There should be specification of any sessions to be worked in the Prison Medical Service and the location of these. Similarly, any sessions working with local authority services (Social Services Department and/ or Probation Service) should be stated.

A multidisciplinary team specifically working in the field of forensic psychiatry should be provided. This should include representatives of the clinical disciplines of psychiatric nursing (including community psychiatric nurses), clinical psychology, social work and occupational therapy. The availability to the team of access to a liaison probation officer is of advantage. It is important that the operational policy for forensic psychiatric services should be agreed by, and be acceptable to, members of the multidisciplinary team.

\section{(b) Relationships with other psychiatric services}

There should be a statement of the relationship between the post and the local psychiatric services generally. Experience has shown that, with the closure of mental hospitals and the in-patient care of patients being provided increasingly in district general hospital units, groups of difficult patients emerge who, while not requiring the security of a special hospital or regional secure unit, nonetheless require special provision. It is likely that general adult psychiatrists will and should look to forensic psychiatrists to advise on the treatment of some such patients. Any special provision made locally for the care of these patients should be described, in order that the consultant forensic psychiatrist's responsibility for such patients, if any, is made explicit.

Similarly, the relationship between the consultant forensic psychiatrist and child and adolescent and mental handicap services should also be described, in particular in relation to the small number of adolescents who may require to be cared for in a secure setting and the small number of the mentally handicapped who require such provision as a result of their challenging behaviours.

\section{Teaching and research}

The pressure to meet service requirements in forensic psychiatry has meant in recent years that growth in National Health Service posts has far outstripped academic development. The provision of at least one session per week for research is to be recommended.

Because of the comparative rarity of forensic psychiatrists, there will in most posts be heavy demands for educational and training services. These commitments should be specified. This will include, in particular, responsibility for the training of senior registrars who may be attached on a rotational basis, be the incumbents of jointly approved posts or undergoing higher professional training in forensic psychiatry. Registrars spending periods of up to six months as part of general professional rotational training schemes will also require teaching and supervision.

Any university status associated with the post should be stated.

\section{Administration}

Any responsibility for the development and monitoring of the local forensic psychiatric service should be stated. This should include any sessional commitment to clinical audit.

The consultant forensic psychiatrist should have a place in the local administrative and management structure.

Adequate secretarial assistance (at least of higher clerical officer grade) and necessary office accommodation and physical amenities must be available for both in-patient and out-patient areas of work.

\section{Approved by the Court of Electors \\ December 1990}

